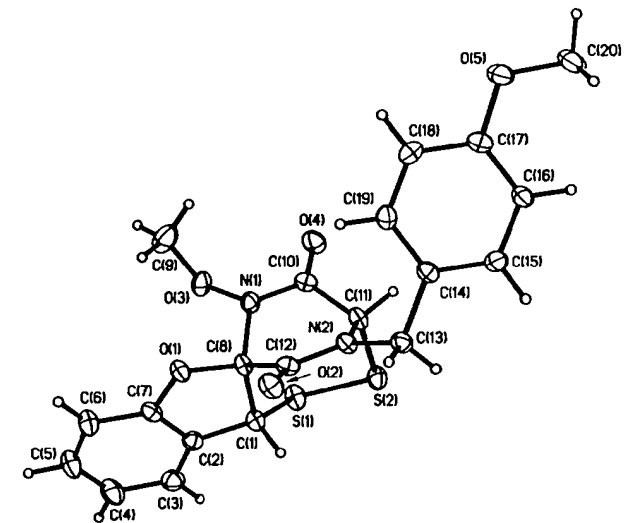

Fig. 1. The structure of (1) with atomic labeling scheme. $50 \%$ probability thermal ellipsoids.
(1), the angles about each $S$ atom are significantly smaller than in (2) [99.7 (1) at $\mathrm{S}(1), 100 \cdot 4(1)^{\circ}$ at $\mathrm{S}(2)$, 100.5 and $101.7^{\circ}$ at analogous atoms in (2)], and the disulfide bond is significantly longer in (1) $[2 \cdot 056(1) \AA]$ than in (2) $(2 \cdot 025 \AA)$.

We acknowledge the support of the US National Science Foundation for the purchase of the Nicolet $R 3 m$ diffractometer and computing system.

\section{References}

Sakata, K., Maruyama, M., Uzawa, J., Sakurai, A., Lu, H. S. M. \& Clardy, J. (1987). Tetrahedron Lett. 28, 5607-5610. SHELDRICK, G. M. (1985). SHELTXTL Users Manual. Revision 5.1. Nicolet XRD Corporation, Madison, Wisconsin, USA.

Acta Cryst. (1991). C47, 1563-1566

\title{
Structure of $18^{\prime}$-Epivinblastine
}

\author{
By Vincent M. Lynch, Andrew Stamford, Philip Magnus and Brian E. Davis \\ Department of Chemistry and Biochemistry, University of Texas at Austin, Austin, TX 78712, USA
}

(Received 1 October 1990; accepted 2 January 1991)

\begin{abstract}
Methyl $\left\{3 \mathrm{a} R-\left[3 \mathrm{a} \alpha, 4 \beta, 5 \beta, 5 \mathrm{a} \beta, 9\left(3 R^{*},-\right.\right.\right.$ $\left.\left.\left.5 S^{*}, 7 R^{*}, 9 R^{*}\right), 10 \mathrm{~b} R, 13 \mathrm{a} \alpha\right]\right\}-4-($ acetyloxy)-3a-ethyl9-[5-ethyl-1,4,5,6,7,8,9,10-octahydro-5-hydroxy-9(methoxycarbonyl)-2H-3,7-methanoazacycloundecino[5,4-b]indol-9-yl]-3a,4,5,5a,6,11,12,13a-octahydro-5-hydroxy-8-methoxy-6-methyl- $1 \mathrm{H}$-indolizino$[8,1-c, d]$ carbazole-5-carboxylate methanol solvate, $\mathrm{C}_{46} \mathrm{H}_{58} \mathrm{~N}_{4} \mathrm{O}_{9} .2 \mathrm{CH}_{3} \mathrm{OH}(1), M_{r}=875 \cdot 07$, monoclinic, $P 2_{1}, \quad a=10.2759(12), \quad b=22.353(3), \quad c=$ $10.4051(12) \AA, \beta=106 \cdot 502(9)^{\circ}, V=2291 \cdot 6(5) \AA^{3}$, $Z=2, \quad D_{x}=1.27 \mathrm{~g} \mathrm{~cm}^{-3}$, Mo $K \alpha$ radiation, $\lambda=$ $0.7107 \AA, \quad \mu=0.8397 \mathrm{~cm}^{-1}, \quad F(000)=940, \quad T=$ $198 \mathrm{~K}, R=0.0470$ for 2751 reflections, $F_{o} \geq 4 \sigma\left(F_{o}\right)$. The $C$ ring of the vindoline moiety is in the boat conformation with the hydroxy group and the tertiary $\mathrm{N}$ in the bowsprit positions resulting in a fairly short intramolecular hydrogen-bonding interaction. The relevant parameters for $\mathrm{O} 3-\mathrm{H} 3 \cdots \mathrm{N} 9$ are $\mathrm{O} \cdots \mathrm{N}$ $2.651(6), \mathrm{H} \cdots \mathrm{N} 1.94(5) \AA$ and $\mathrm{O}-\mathrm{H} \cdots \mathrm{N} 147(5)^{\circ}$. The $D$ and $E$ rings are in the sofa and envelope conformations, respectively. The piperidine ring of the catharanthine portion of the molecule assumes the chair conformation while the conformation of the azacyclononene ring is a boat-chair. An intramolecular hydrogen bond between the indolino $\mathrm{NH}$ of the catharanthine moiety and methoxy $\mathrm{O}(\mathrm{O} 25)$ of the vindoline moiety is also observed. The relevant parameters for $\mathrm{N} 16^{\prime}-\mathrm{H} 16^{\prime} \cdots \mathrm{O} 25$ are $\mathrm{N} \cdots \mathrm{O}$ $2 \cdot 827(6), \mathrm{H} \cdots \mathrm{N} 2 \cdot 14$ (6) $\AA$ and $\mathrm{O}-\mathrm{H}^{\cdots} \cdot \mathrm{N} 136(5)^{\circ}$.
\end{abstract}

0108-2701/91/071563-04\$03.00
Experimental. (1) was prepared during the course of a synthesis of the clinically important antitumor alkaloid vinblastine (Moncrief \& Lipscomb, 1965; Magnus, Stamford \& Ladlow, 1991). The absolute

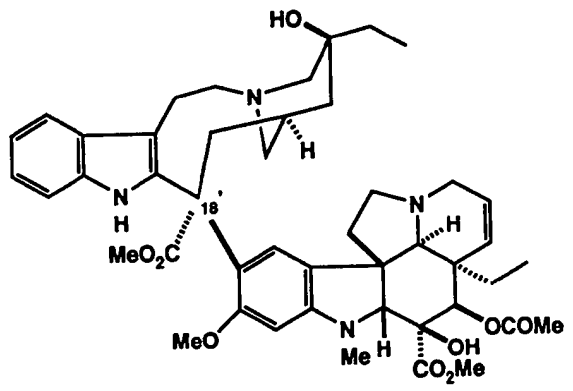

(1)

configuration of (1) was assigned on the basis of internal comparison to the vindoline moiety (Moncrief \& Lipscomb, 1966). Colorless crystals were obtained by slow evaporation of a methanolwater solution. The data crystal was separated from a cluster of smaller crystals and had approximate dimensions $0.17 \times 0.34 \times 0.43 \mathrm{~mm}$. The data were collected on a Nicolet $P 3$ diffractometer using a graphite monochromator and equipped with a Nicolet LT-2 low-temperature device. The lattice parameters were obtained by the least-squares refinement of 40 reflections with $18 \cdot 2<2 \theta<21 \cdot 2^{\circ}$.

(C) 1991 International Union of Crystallography 
The data were collected from 4.0 to $50^{\circ}$ in $2 \theta$ using the $\omega$-scan technique, with a $1.2^{\circ}$ scan range at $2-5^{\circ} \mathrm{min}^{-1}$. 8740 reflections were measured, of which 4278 were unique $\left(R_{\text {int }}=0.0195\right)$, where $h$ ranged from $-12 \rightarrow 12, k$ from $0 \rightarrow 26$ and $I$ from $-12 \rightarrow 12$. Four reflections $(112,1 \overline{5} \overline{1}, \overline{2} \overline{2} 2,1 \overline{4} 0)$ were remeasured every 96 reflections to monitor instrument and crystal stability. A smoothed curve of the intensities of these check reflections was used to scale the data. The scaling factor ranged from 1.00-1.09. The data were also corrected for Lp effects but not absorption. Reflections having $F_{o}<4 \sigma\left(F_{o}\right)$ were considered unobserved (1527 reflections). Data reduction and decay correction were performed using the Nicolet XRD SHELXTL-Plus (Sheldrick, 1988) software package. The structure was solved by Patterson search methods using PATSEE (Egert \& Sheldrick, 1985 ) and refined by full-matrix least squares (Sheldrick, 1988) with anisotropic thermal parameters for the non- $\mathrm{H}$ atoms. Most $\mathrm{H}$ atoms were located from a $\Delta F$ map and refined with isotropic thermal parameters. The $\mathrm{H}$ atoms on $\mathrm{C}^{\prime}, \mathrm{C}^{\prime} 1^{\prime}$, C23', C20, C21, C24, C25, C26 and C27 did not refine well and were idealized (C-H $0.96 \AA$ ) with isotropic $U$ refined. The $\mathrm{H}$ atoms on the solvent methanol atoms $(\mathrm{O} 1 B, \mathrm{C} 2 B, \mathrm{C} 2 A)$ were idealized with isotropic $U$ fixed at $1.2 \times U_{\text {eq }}$ of the relevant atom. The $\mathrm{H}$ atom on $\mathrm{O} 1 A$ (methanol $\mathrm{O}$ ) could not be located and was omitted from refinement. $\sum w\left(\left|F_{o}\right|-\left|F_{c}\right|\right)^{2}$ was minimized where $w=1 /\left[\sigma\left(F_{o}\right)\right]^{2}$ and $\sigma\left(F_{o}\right)=0.5 k I^{-1 / 2}\left\{[\sigma(I)]^{2}+(0.02 I)^{2}\right\}^{1 / 2}$. The intensity, $I$, is given by $\left(I_{\text {peak }}-I_{\text {background }}\right) \times($ scan rate); where 0.02 is a factor to downweight intense reflections and to account for instrument instability and $k$ is the correction due to Lp effects and decay. $\sigma(I)$ was estimated from counting statistics; $\sigma(I)=$ $\left[\left(I_{\text {peak }}+I_{\text {background }}\right)^{1 / 2} \times(\right.$ scan rate $\left.)\right] .725$ independent parameters were refined in blocks of 362 and 364 parameters (a single overall scale factor was refined in all cycles) to a final $R=0.0470$ for 2751 reflections, with $w R=0.0405$ and goodness of fit, $S=$ $1 \cdot 118$. The $R$ value for all data was $0 \cdot 0845$, with $w R$ $=0.0488$. Max. $|\Delta / \sigma|<0.1$ in the final refinement cycle and the min. and max. peaks in the final difference electron density map were -0.22 , $0.22 \mathrm{e} \AA^{-3}$, respectively. The origin was defined along the $2_{1}$ screw axis by the atom positions of the block not being refined. Neutral atom scattering factors for the non- $\mathrm{H}$ atoms were taken from Cromer \& Mann (1968), with the anomalousdispersion corrections taken from the work of Cromer \& Liberman (1970). The scattering factors for the $\mathrm{H}$ atom were obtained from Stewart, Davidson \& Simpson (1965). Values used to calculate the linear absorption coefficient are from International Tables for X-ray Crystallography (1974, Vol. IV, p. 55). All figures were generated using
Table 1. Fractional coordinates and equivalent isotropic thermal parameters $\left(\AA^{2}\right)$ for the non-H atoms of $(1)$

$$
U_{\mathrm{eq}}=(1 / 3) \sum_{i} \sum_{j} U_{i j} a_{i}^{*} a_{j}^{*} \mathbf{a}_{i} \cdot \mathbf{a}_{j} .
$$

\begin{tabular}{|c|c|c|c|c|}
\hline & $x$ & $y$ & $z$ & $U_{\mathrm{eq}}$ \\
\hline $\mathrm{Cl}^{\prime}$ & $-0 \cdot 1422(5)$ & $0.1507(2)$ & $0.4400(6)$ & $0.026(2)$ \\
\hline $\mathrm{C} 2^{\prime}$ & $-0.2464(6)$ & $0.1122(2)$ & $0.4832(6)$ & $0.033(2)$ \\
\hline$C 3^{\prime}$ & $-0.3825(6)$ & $0.1446(3)$ & $0.4737(6)$ & $0.039(2)$ \\
\hline$C 4^{\prime}$ & $-0.3836(5)$ & $0.1901(3)$ & $0 \cdot 5805(6)$ & $0.034(2)$ \\
\hline$O 4^{\prime}$ & $-0.3223(5)$ & $0.2448(2)$ & $0.5492(5)$ & $0.042(2)$ \\
\hline $\mathrm{C} 5^{\prime}$ & $-0.2988(7)$ & $0 \cdot 1680(3)$ & $0.7192(6)$ & $0.038(2)$ \\
\hline $\mathrm{N} 6^{\prime}$ & $-0.1716(5)$ & $0 \cdot 1440(2)$ & $0.7114(4)$ & $0.038(2)$ \\
\hline $\mathrm{Cl}^{\prime}$ & $-0.0461(7)$ & $0.1526(3)$ & $0.8120(6)$ & $0.043(3)$ \\
\hline $\mathrm{C} 8^{\prime}$ & $0.0677(7)$ & $0.1743(3)$ & $0.7532(7)$ & $0.038(2)$ \\
\hline $\mathrm{C} 9^{\prime}$ & $0 \cdot 1317(5)$ & $0 \cdot 1276(2)$ & $0 \cdot 6864(5)$ & $0.028(2)$ \\
\hline $\mathrm{C} 10^{\prime}$ & $0 \cdot 2555(5)$ & $0.0990(3)$ & $0.7580(5)$ & $0.030(2)$ \\
\hline $\mathrm{Cll}^{\prime}$ & $0 \cdot 3405(6)$ & $0.1035(3)$ & $0 \cdot 8882(6)$ & $0.040(2)$ \\
\hline $\mathrm{C} 12^{\prime}$ & $0.4571(7)$ & $0.0687(3)$ & $0.9267(6)$ & $0.049(3)$ \\
\hline $\mathrm{Cl}^{\prime}$ & $0.4924(7)$ & $0.0299(3)$ & $0.8381(6)$ & $0.044(3)$ \\
\hline $\mathrm{C} 14^{\prime}$ & $0.4100(6)$ & $0.0244(3)$ & $0.7087(6)$ & $0.041(3)$ \\
\hline $\mathrm{Cls}^{\prime}$ & $0.2929(5)$ & $0.0591(2)$ & $0.6703(5)$ & $0.030(2)$ \\
\hline $\mathrm{N} 16^{\prime}$ & $0 \cdot 1940(5)$ & $0.0632(2)$ & $0 \cdot 5504(5)$ & $0.030(2)$ \\
\hline $\mathrm{Cl} 7^{\prime}$ & $0.0962(5)$ & $0 \cdot 1043(2)$ & $0.5597(5)$ & $0.026(2)$ \\
\hline $\mathrm{C} 18^{\prime}$ & $-0.0118(5)$ & $0 \cdot 1184(2)$ & $0.4279(5)$ & $0.026(2)$ \\
\hline $\mathrm{C} 19^{\prime}$ & $-0 \cdot 1903(7)$ & $0.0910(3)$ & $0.6275(6)$ & $0.038(2)$ \\
\hline $\mathrm{C} 20^{\prime}$ & $-0.5291(7)$ & $0.2051(3)$ & $0.5796(7)$ & $0.047(3)$ \\
\hline $\mathrm{C} 21^{\prime}$ & $-0.5436(7)$ & $0 \cdot 2476(4)$ & $0.6864(7)$ & $0.064(3)$ \\
\hline $\mathrm{C} 22^{\prime}$ & -0.0678 & 0.0607 (3) & $0.3504(5)$ & $0.032(2)$ \\
\hline $\mathrm{O} 22^{\prime}$ & $-0.0577(4)$ & $0.0115(2)$ & $0 \cdot 3989(4)$ & $0.038(2)$ \\
\hline $\mathrm{O} 23^{\prime}$ & $-0.1376(4)$ & $0.0736(2)$ & $0.2246(4)$ & $0.039(2)$ \\
\hline $\mathrm{C} 23^{\prime}$ & $-0.1932(7)$ & $0.0225(3)$ & $0 \cdot 1425(7)$ & $0.054(3)$ \\
\hline N1 & $0.2596(4)$ & $0.2680(2)$ & $0.1376(4)$ & $0.031(2)$ \\
\hline $\mathrm{C} 2$ & $0.2312(5)$ & $0.3291(2)$ & $0.1778(5)$ & $0.024(2)$ \\
\hline C3 & $0 \cdot 1664(5)$ & $0.3685(2)$ & $0.0519(5)$ & $0.026(2)$ \\
\hline O3 & $0 \cdot 1729(4)$ & $0.4299(2)$ & $0.0860(4)$ & $0.031(2)$ \\
\hline C4 & $0.0233(5)$ & $0.3457(2)$ & $-0.0142(5)$ & $0.025(2)$ \\
\hline $\mathrm{O} 4$ & $-0.0291(3)$ & $0.3806(2)$ & $-0.1351(3)$ & 0.029 (1) \\
\hline C5 & $-0.0767(5)$ & $0.3479(2)$ & $0.0736(5)$ & $0.026(2)$ \\
\hline C6 & $-0.1739(6)$ & $0.3995(3)$ & $0.0382(7)$ & $0.038(2)$ \\
\hline $\mathrm{C} 7$ & $-0.1836(6)$ & $0.4437(3)$ & $0 \cdot 1180(6)$ & $0.040(3)$ \\
\hline $\mathrm{C} 8$ & $-0.0856(6)$ & $0.4517(3)$ & $0.2535(7)$ & $0.039(3)$ \\
\hline N9 & $0.0347(4)$ & $0.4151(2)$ & $0.2634(4)$ & $0.030(2)$ \\
\hline $\mathrm{ClO}$ & $0.1367(7)$ & $0.4142(3)$ & $0.3955(7)$ & $0.045(3)$ \\
\hline $\mathrm{Cll}$ & $0.1968(6)$ & $0.3514(3)$ & $0.4101(6)$ & $0.032(2)$ \\
\hline $\mathrm{Cl} 2$ & $0.1382(6)$ & $0 \cdot 3210(2)$ & $0 \cdot 2739(5)$ & $0.028(2)$ \\
\hline $\mathrm{C} 13$ & $0 \cdot 1279(5)$ & $0.2535(2)$ & $0.2816(5)$ & $0.026(2)$ \\
\hline $\mathrm{C} 14$ & $0.0597(5)$ & $0.2192(3)$ & $0 \cdot 3527(5)$ & 0.029 (2) \\
\hline $\mathrm{C} 15$ & $0.0612(5)$ & $0.1569(2)$ & $0 \cdot 3460(5)$ & $0.024(2)$ \\
\hline C16 & $0.1391(5)$ & $0.1305(2)$ & $0.2685(5)$ & $0.026(2)$ \\
\hline $\mathrm{Cl} 7$ & $0 \cdot 2084(6)$ & $0 \cdot 1647(2)$ & $0 \cdot 1990(5)$ & $0.030(2)$ \\
\hline $\mathrm{C} 18$ & $0.2012(5)$ & $0.2262(2)$ & $0 \cdot 2050(5)$ & $0.025(2)$ \\
\hline $\mathrm{Cl9}$ & $0.0002(5)$ & $0 \cdot 3525(2)$ & $0 \cdot 2235(5)$ & $0.026(2)$ \\
\hline $\mathrm{C} 20$ & $-0.1593(6)$ & $0 \cdot 2882(3)$ & $0.0443(6)$ & 0.038 \\
\hline $\mathrm{C} 21$ & $-0.2678(6)$ & $0 \cdot 2783(3)$ & $0.1163(8)$ & $0.054(3)$ \\
\hline $\mathrm{C} 22$ & $-0.0819(6)$ & $0.3502(3)$ & $-0.2498(6)$ & $0.036(2)$ \\
\hline $\mathrm{O} 22$ & $-0 \cdot 1093(4)$ & $0.2984(2)$ & $-0.2551(4)$ & $0.049(2)$ \\
\hline $\mathrm{C} 23$ & $0.2494(6)$ & $0.3617(3)$ & $-0.0472(6)$ & $0.033(2)$ \\
\hline $\mathrm{O} 23$ & $0.2191(4)$ & $0.3303(2)$ & $-0 \cdot 1456(4)$ & $0.047(2)$ \\
\hline $\mathrm{C} 24$ & $0.4441(7)$ & $0.3920(3)$ & $-0 \cdot 1045(7)$ & $0.054(3)$ \\
\hline $\mathrm{O} 24$ & $0.3610(4)$ & $0.3949(2)$ & $-0.0143(4)$ & $0.039(2)$ \\
\hline $\mathrm{C} 25$ & $0 \cdot 1678(8)$ & $0.0401(3)$ & $0 \cdot 1550(6)$ & $0.053(3)$ \\
\hline $\mathrm{O} 25$ & $0.1448(4)$ & $0.0692(2)$ & $0.2694(3)$ & $0.034(1)$ \\
\hline C26 & $-0.1028(8)$ & $0.3906(3)$ & $-0.3671(6)$ & 0.061 (3) \\
\hline $\mathrm{C} 27$ & $0.3915(6)$ & $0.2546(3)$ & $0 \cdot 1216(7)$ & $0.051(3)$ \\
\hline $\mathrm{Ol} A$ & $0.5555(5)$ & $0.3497(2)$ & $0.4572(5)$ & $0.076(2)$ \\
\hline $\mathrm{C} 2 A$ & $0.605(1)$ & $0.3639(4)$ & $0.3557(9)$ & $0 \cdot 100(4)$ \\
\hline $\mathrm{O} 1 B$ & $0.5871(7)$ & $0.4334(3)$ & $0.6620(7)$ & $0 \cdot 125(3)$ \\
\hline$C 2 B$ & $0.495(1)$ & $0.4811(4)$ & $0.6281(9)$ & $0 \cdot 117(5)$ \\
\hline
\end{tabular}

SHELXTL-Plus (Sheldrick, 1988). The positional and thermal parameters for the non- $\mathrm{H}$ atoms are listed in Table $1 .^{*}$ The bond lengths and angles are

* Lists of structure factors, anisotropic thermal parameters, bond distances and angles involving $\mathrm{H}$ atoms, torsion angles, $\mathrm{H}$-bonding interactions and $\mathrm{H}$-atom parameters and a unit-cell packing diagram have been deposited with the British Library Document Supply Centre as Supplementary Publication No. SUP 53891 (34 pp.). Copies may be obtained through The Technical Editor, International Union of Crystallography, 5 Abbey Square, Chester CH1 2HU, England. 
Table 2. Bond lengths $(\AA)$ and angles $\left(^{\circ}\right)$ for the non- $\mathrm{H}$ atoms of (1)

\begin{tabular}{|c|c|c|c|c|}
\hline 1 & 2 & 3 & $1-2$ & $1-2-3$ \\
\hline $\mathrm{C} 2^{\prime}$ & $\mathrm{Cl}^{\prime}$ & $\mathrm{Cl} 8^{\prime}$ & $1.537(9)$ & $116.8(4)$ \\
\hline $\mathrm{C} 18^{\prime}$ & $\mathrm{Cl}^{\prime}$ & & $1.559(8)$ & \\
\hline C3' & $\mathrm{C} 2^{\prime}$ & $\mathrm{C} 19^{\prime}$ & $1.553(8)$ & $106.8(5)$ \\
\hline$C 3^{\prime}$ & $\mathrm{C} 2^{\prime}$ & $\mathrm{Cl}^{\prime}$ & & $1 / 4 \cdot 2(4)$ \\
\hline $\mathrm{C}^{\prime} 9^{\prime}$ & $\mathrm{C} 2$ & $\mathrm{Cl}^{\prime}$ & $1.523(8)$ & $111 \cdot 3(5)$ \\
\hline $\mathrm{C} 4^{\prime}$ & $\mathrm{C}^{\prime}$ & $\mathrm{C} 2^{\prime}$ & $1.509(8)$ & $117.5(4)$ \\
\hline $\mathrm{O}^{\prime}$ & $\mathrm{C}^{\prime}$ & $\mathrm{Cs}^{\prime}$ & $1.455(7)$ & $108 \cdot 3(4)$ \\
\hline $\mathrm{O}^{\prime}$ & $\mathrm{C}^{\prime}$ & $\mathrm{C} 20^{\prime}$ & & $107.4(5)$ \\
\hline $\mathrm{O}^{\prime}$ & $\mathrm{C}^{\prime}$ & $\mathrm{C}^{\prime}$ & & $107.9(5)$ \\
\hline$C 5^{\prime}$ & $\mathrm{C}^{\prime}$ & $\mathrm{C} 20^{\prime}$ & $1.539(8)$ & $111.6(6)$ \\
\hline $\mathrm{Cs}^{\prime}$ & $\mathrm{C}^{\prime}$ & $\mathrm{C}^{\prime}$ & & $110 \cdot 7(5)$ \\
\hline $\mathrm{C} 20^{\prime}$ & C4' & $\mathrm{C} 3^{\prime}$ & $1.529(9)$ & $110.8(5)$ \\
\hline N6' & $\mathrm{C} 5^{\prime}$ & $C 4^{\prime}$ & $1.437(9)$ & $110.4(5)$ \\
\hline $\mathrm{C}^{\prime}$ & N6 & $\mathrm{C} 19^{\circ}$ & $1.424(7)$ & $119 \cdot 4(5)$ \\
\hline $\mathrm{C}^{\prime}$ & N6 ${ }^{\prime}$ & $\mathrm{C} 5$ & & $123.8(5)$ \\
\hline $\mathrm{C} 19^{\prime}$ & N6 & $\mathrm{C}^{\prime}$ & $1.451(7)$ & $111.8(5)$ \\
\hline C $8^{\circ}$ & $\mathrm{C}^{\prime}$ & N6 ${ }^{\prime}$ & $1.55(1)$ & $112.4(5)$ \\
\hline $\mathrm{C} 9^{\prime}$ & $\mathrm{C}^{\prime}$ & $\mathrm{C}^{\prime}$ & $1.505(9)$ & $116 \cdot 3(5)$ \\
\hline $\mathrm{C} 10^{\circ}$ & $\mathrm{C}^{\prime}$ & $\mathrm{C} 17^{\prime}$ & $1.430(7)$ & $106.6(5)$ \\
\hline $\mathrm{ClO}^{\prime}$ & $\mathrm{C}^{\prime}$ & $\mathrm{C}^{\prime}$ & & $120.7(5)$ \\
\hline $\mathrm{C} 17^{\prime}$ & $\mathrm{C} 9^{\prime}$ & $\mathrm{C}^{\prime}$ & $1 \cdot 367(7)$ & $132 \cdot 6(5)$ \\
\hline $\mathrm{C}^{\circ} \mathrm{I}^{\circ}$ & $\mathrm{C} 10^{\prime}$ & $\mathrm{C}^{\prime} \mathrm{s}^{\prime}$ & $1.392(7)$ & $117.8(5)$ \\
\hline $\mathrm{Cll} 1^{\circ}$ & $\mathrm{C} 10^{\prime}$ & $C 9^{\prime}$ & & $134.0(6)$ \\
\hline $\mathrm{Cl}^{\prime}$ & $\mathrm{ClO}^{\prime}$ & $\mathrm{C} 9^{\prime}$ & $1.404(8)$ & $108 \cdot 2(4)$ \\
\hline $\mathrm{C} 12^{\prime}$ & $\mathrm{Cll}$ & $\mathrm{C} 10^{\prime}$ & $1.389(9)$ & $119 \cdot 4(6)$ \\
\hline $\mathrm{C} 13^{\prime}$ & $\mathrm{C} 12^{\prime}$ & $\mathrm{Cll}$ & $1.39(1)$ & $121.7(5)$ \\
\hline C14' & $\mathrm{C} 13^{\prime}$ & $\mathrm{C} 12^{\prime}$ & $1.377(8)$ & $120.1(6)$ \\
\hline $\mathrm{C} 15^{\prime}$ & C14' & $\mathrm{Cl}^{\circ}$ & $1.391(8)$ & $118.2(6)$ \\
\hline N16 & $\mathrm{C} 15^{\prime}$ & $\mathrm{ClO}^{\circ}$ & $1.370(6)$ & $106 \cdot 3(5)$ \\
\hline $\mathrm{N} 16^{\prime}$ & $\mathrm{C} 15^{\prime}$ & $\mathrm{Cl} 4^{\prime}$ & & $131 \cdot 0(5)$ \\
\hline $\mathrm{C} 10^{\prime}$ & $\mathrm{C} 15^{\prime}$ & $\mathrm{C} 14^{\prime}$ & & $122.8(5)$ \\
\hline $\mathrm{Cl} 7^{\prime}$ & $\mathrm{N} 16^{\prime}$ & $\mathrm{C} 15^{\prime}$ & $1 \cdot 386(7)$ & $110 \cdot 2(5)$ \\
\hline $\mathrm{C} 18^{\prime}$ & $\mathrm{C} 17^{\prime}$ & $\mathrm{C}^{\prime}$ & $1.531(6)$ & $135.4(5)$ \\
\hline $\mathrm{C} 18^{\prime}$ & $\mathrm{C} 17^{\prime}$ & $\mathrm{N} 16^{\prime}$ & & $115.4(5)$ \\
\hline $\mathrm{C} 9^{\prime}$ & $\mathrm{Cl} 7^{\prime}$ & $\mathrm{N} 16^{\prime}$ & & $108 \cdot 6(4)$ \\
\hline $\mathrm{C} 22^{\prime}$ & $\mathrm{C} 18^{\prime}$ & $\mathrm{C} 15$ & $1 \cdot 543(7)$ & $110 \cdot 1(4)$ \\
\hline $\mathrm{C} 22^{\prime}$ & $\mathrm{Cl}^{\prime}$ & $\mathrm{Cl}^{\prime}$ & & $103 \cdot 1(4)$ \\
\hline $\mathrm{C} 22^{\prime}$ & $\mathrm{Cl} 8^{\prime}$ & $\mathrm{C} 17^{\prime}$ & & $111 \cdot 3(4)$ \\
\hline $\mathrm{C} 15$ & $\mathrm{C} 18^{\prime}$ & $\mathrm{Cl}^{\prime}$ & $1.547(8)$ & $110.4(4)$ \\
\hline $\mathrm{Cl5}$ & $\mathrm{C} 18^{\prime}$ & $\mathrm{C} 17^{\prime}$ & & $105 \cdot 5(4)$ \\
\hline $\mathrm{Cl}^{\prime}$ & C $18^{\prime}$ & $\mathrm{Cl}^{\prime}$ & & $116.4(5)$ \\
\hline $\mathrm{C} 2^{\prime}$ & $\mathrm{C} 19^{\prime}$ & $N 6^{\prime}$ & & $106 \cdot 7(4)$ \\
\hline $\mathrm{C} 2 !^{\prime}$ & $\mathrm{C} 20^{\circ}$ & $\mathrm{C} 4^{\prime}$ & $1.50(1)$ & $115.9(5)$ \\
\hline $\mathrm{O} 22^{\prime}$ & $\mathrm{C} 22^{\prime}$ & O23' & $1 \cdot 202(7)$ & $124 \cdot 5(5)$ \\
\hline $\mathrm{O} 22^{\prime}$ & $\mathrm{C} 22^{\prime}$ & $\mathrm{C} 18^{\prime}$ & & $124 \cdot 8(5)$ \\
\hline $\mathrm{O} 23^{\prime}$ & $\mathrm{C} 22^{\prime}$ & $\mathrm{C} 18^{\prime}$ & $1.333(6)$ & $110 \cdot 5(5)$ \\
\hline $\mathrm{C} 23^{\prime}$ & $\mathrm{O} 23^{\prime}$ & $\mathrm{C} 22^{\prime}$ & $1.443(7)$ & $115 \cdot 1(4)$ \\
\hline $\mathrm{C} 2$ & N1 & $\mathrm{C} 18$ & $1.482(7)$ & $109 \cdot 1(5)$ \\
\hline $\mathrm{C} 18$ & N1 & $\mathrm{C} 27$ & $1.401(7)$ & $118.0(4)$ \\
\hline $\mathrm{C} 27$ & N1 & $\mathrm{C} 2$ & $1.443(8)$ & $119.4(4)$ \\
\hline C3 & $\mathrm{C} 2$ & $\mathrm{C} 12$ & $1.561(7)$ & $114.0(4)$ \\
\hline C3 & $\mathrm{C} 2$ & $\mathrm{~N} 1$ & & $110.7(4)$ \\
\hline $\mathrm{C} 12$ & $\mathrm{C} 2$ & NI & $1.578(9)$ & $106 \cdot 0(4)$ \\
\hline $\mathrm{O} 3$ & $\mathrm{C} 3$ & $\mathrm{C} 4$ & $1.414(6)$ & $114 \cdot 1(4)$ \\
\hline $\mathrm{O} 3$ & $\mathrm{C} 3$ & $\mathrm{C} 23$ & & $105 \cdot 7(4)$ \\
\hline O3 & $\mathrm{C} 3$ & $\mathrm{C} 2$ & & $110.9(4)$ \\
\hline C4 & $\mathrm{C} 3$ & $\mathrm{C} 23$ & $1.523(7)$ & $108.4(4)$ \\
\hline C4 & $\mathrm{C} 3$ & $\mathrm{C} 2$ & & $108 \cdot 5(4)$ \\
\hline $\mathrm{C} 23$ & $\mathrm{C} 3$ & $\mathrm{C} 2$ & $1.520(9)$ & $109 \cdot 0(4)$ \\
\hline O4 & $\mathrm{C} 4$ & C5 & $1.447(6)$ & $110.4(4)$ \\
\hline O4 & C4 & $\mathrm{C} 3$ & & $106.6(4)$ \\
\hline $\mathrm{C} 5$ & $\mathrm{C} 4$ & C3 & $1 \cdot 558(8)$ & $116.0(4)$ \\
\hline $\mathrm{C} 22$ & O4 & $\mathrm{C} 4$ & $1 \cdot 346(6)$ & $117 \cdot 1$ (4) \\
\hline C6 & $\mathrm{C} 5$ & $\mathrm{C} 19$ & $1.502(8)$ & $108 \cdot 1(5)$ \\
\hline C6 & $\mathrm{C} 5$ & $\mathrm{C} 20$ & & $108 \cdot 7(4)$ \\
\hline C6 & C5 & $\mathrm{C} 4$ & & $112.5(5)$ \\
\hline $\mathrm{C} 19$ & $\mathrm{C} 5$ & $\mathrm{C} 20$ & $1.539(7)$ & $110 \cdot 5(4)$ \\
\hline $\mathrm{C} 19$ & $\mathrm{C} 5$ & $\mathrm{C} 4$ & & $111 \cdot 3(4)$ \\
\hline $\mathrm{C} 20$ & $\mathrm{C} 5$ & $\mathrm{C} 4$ & $1.563(8)$ & $105 \cdot 8$ (4) \\
\hline $\mathrm{C} 7$ & $\mathrm{C} 6$ & $\mathrm{C} 5$ & $1.312(9)$ & 126.0 \\
\hline $\mathrm{C} 8$ & $C 7$ & C6 & $1.493(8)$ & $122 \cdot 4(6)$ \\
\hline N9 & $\mathrm{C} 8$ & $\mathrm{C} 7$ & $1.460(8)$ & $109 \cdot 3(5)$ \\
\hline $\mathrm{ClO}$ & N9 & $\mathrm{C} 19$ & $1.473(7)$ & $107 \cdot 5(4)$ \\
\hline $\mathrm{ClO}$ & N9 & $\mathrm{C} 8$ & & $115.9(5)$ \\
\hline $\mathrm{C} 19$ & N9 & $\mathrm{C} 8$ & $1.474(6)$ & $112 \cdot 3(4)$ \\
\hline $\mathrm{C} 11$ & $\mathrm{ClO}$ & N9 & $1.524(8)$ & $105 \cdot 5(5)$ \\
\hline $\mathrm{Cl} 2$ & $\mathrm{C} 11$ & $\mathrm{ClO}$ & $1.533(7)$ & $105.9(4)$ \\
\hline $\mathrm{C} 13$ & $\mathrm{C} 12$ & $\mathrm{C} 19$ & $1.517(7)$ & $113.8(4)$ \\
\hline $\mathrm{C} 13$ & $\mathrm{Cl} 2$ & $\mathrm{C} 2$ & & $102 \cdot 2(4)$ \\
\hline $\mathrm{C} 13$ & $\mathrm{C} 12$ & $\mathrm{Cll}$ & & $114.0(4)$ \\
\hline $\mathrm{C} 19$ & $\mathrm{C} 12$ & $\mathrm{C} 2$ & $1.536(7)$ & $113.4(4)$ \\
\hline $\mathrm{C} 19$ & $\mathrm{C} 12$ & $\mathrm{C} 11$ & & $101 \cdot 2(4)$ \\
\hline $\mathrm{C} 2$ & $\mathrm{Cl} 2$ & $\mathrm{Cll}$ & & $1127(5)$ \\
\hline $\mathrm{Cl} 4$ & $\mathrm{C} 13$ & $\mathrm{C} 18$ & $1.385(8)$ & $120 \cdot 3(5)$ \\
\hline
\end{tabular}

Table 2 (cont.)

\begin{tabular}{|c|c|c|c|c|}
\hline 1 & 2 & 3 & $1-2$ & $1-2-3$ \\
\hline $\mathrm{C} 14$ & $\mathrm{C} 13$ & $\mathrm{C} 12$ & & $129 \cdot 2(5)$ \\
\hline $\mathrm{C} 18$ & $\mathrm{C} 13$ & $\mathrm{C} 12$ & $1.384(8)$ & $110.5(5)$ \\
\hline $\mathrm{C} 15$ & $\mathrm{C} 14$ & $\mathrm{Cl} 3$ & $1.395(7)$ & $120 \cdot 8(5)$ \\
\hline $\mathrm{C} 16$ & $\mathrm{C} 15$ & $\mathrm{C} 18^{\prime}$ & $1.416(8)$ & $121 \cdot 5(4)$ \\
\hline $\mathrm{C} 16$ & $\mathrm{C} 15$ & $\mathrm{C} 14$ & & $117 \cdot 4(5)$ \\
\hline $\mathrm{C} 18^{\circ}$ & $\mathrm{C} 15$ & $\mathrm{C} 14$ & & $120.9(5)$ \\
\hline $\mathrm{Cl} 7$ & $\mathrm{Cl}$ & $\mathrm{O} 25$ & $1.381(9)$ & $121.9(5)$ \\
\hline $\mathrm{C} 17$ & $\mathrm{Cl} 6$ & $\mathrm{C} 15$ & & $121.8(5)$ \\
\hline $\mathrm{O} 25$ & $\mathrm{Cl}$ & $\mathrm{Cl5}$ & $1 \cdot 373(6)$ & $116 \cdot 3(5)$ \\
\hline $\mathrm{C} 18$ & $\mathrm{C} 17$ & $\mathrm{C} 16$ & $1 \cdot 380(7)$ & $119.0(6)$ \\
\hline $\mathrm{N} 1$ & $\mathrm{C} 18$ & C 13 & & $112.1(5)$ \\
\hline $\mathrm{NI}$ & $\mathrm{C} 18$ & $\mathrm{C} 17$ & & $127 \cdot 3(5)$ \\
\hline $\mathrm{C} 13$ & $\mathrm{C} 18$ & $\mathrm{C} 17$ & & $120 \cdot 6(5)$ \\
\hline C5 & $\mathrm{C} 19$ & N9 & & $111 \cdot 3(4)$ \\
\hline C5 & $\mathrm{C} 19$ & $\mathrm{C} 12$ & & $118.2(5)$ \\
\hline N9 & $\mathrm{C} 19$ & $\mathrm{C} 12$ & & $102 \cdot 4(4)$ \\
\hline $\mathrm{C} 21$ & $\mathrm{C} 20$ & C5 & $1.53(1)$ & $117 \cdot 4(5)$ \\
\hline $\mathrm{O} 22$ & $\mathrm{C} 22$ & $\mathrm{C} 26$ & $1 \cdot 188(7)$ & $125 \cdot 2(5)$ \\
\hline $\mathrm{O} 22$ & $\mathrm{C} 22$ & $\mathrm{O} 4$ & & $124 \cdot 3(5)$ \\
\hline $\mathrm{C} 26$ & $\mathrm{C} 22$ & $\mathrm{O} 4$ & $1.484(9)$ & $110.5(5)$ \\
\hline $\mathrm{O} 23$ & $\mathrm{C} 23$ & $\mathrm{O} 24$ & $1 \cdot 208(7)$ & $122 \cdot 7(6)$ \\
\hline $\mathrm{O} 23$ & $\mathrm{C} 23$ & $\mathrm{C} 3$ & & $125 \cdot 0(5)$ \\
\hline O24 & $\mathrm{C} 23$ & C3 & $1 \cdot 326(7)$ & $112 \cdot 3(5)$ \\
\hline $\mathrm{O} 24$ & C24 & & $1.438(9)$ & \\
\hline $\mathrm{C} 23$ & $\mathrm{O} 24$ & $\mathrm{C} 24$ & & $115.9(5)$ \\
\hline $\mathrm{O} 25$ & $\mathrm{C} 25$ & & $1.433(8)$ & \\
\hline $\mathrm{Cl} 6$ & $\mathrm{O} 25$ & $\mathrm{C} 25$ & & $117.7(4)$ \\
\hline $\mathrm{C} 2 \mathrm{~A}$ & $\mathrm{Ol} A$ & & $1.34(1)$ & \\
\hline C2B & O1 $B$ & & $1.40(1)$ & \\
\hline
\end{tabular}

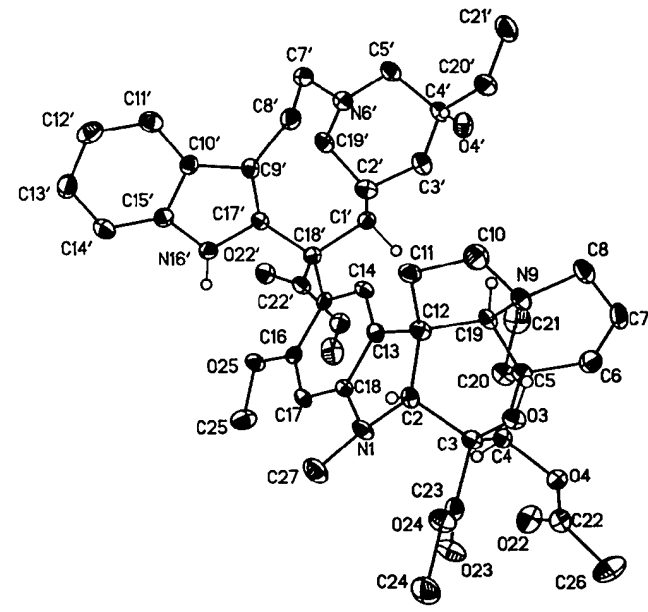

Fig. 1. View of $\mathrm{C}_{46} \mathrm{H}_{58} \mathrm{~N}_{4} \mathrm{O}_{9}$ (1) showing the atom-labeling scheme. Thermal ellipsoids are scaled to the $30 \%$ probability level. Most $\mathrm{H}$ atoms were removed for clarity. $\mathrm{H}$ atoms represented as spheres of arbitrary size. The methanol molecules are not shown.

listed in Table 2. The atom-labeling scheme is shown in Fig. 1. Other computer programs used in this work are listed elsewhere (Gadol \& Davis, 1982).

Related literature. Bisindole derivatives of (1) have been prepared previously (Kutney, Beck, Bylsma, Cook, Cretney, Fuji, Imhof \& Treasurywala, 1975; Langlois, Gueritte, Langlois \& Potier, 1976; Potier, 1980; Kuehne, Zebovitz, Bornmann \& Marko, 1987; Kuehne \& Bornmann, 1989). 
The National Institutes of the Health (GM 29801 to PM) are thanked for their support of this research.

\section{References}

Cromer, D. T. \& Liberman, D. (1970). J. Chem. Phys. 53, 1891-1898.

Cromer, D. T. \& ManN, J. B. (1968). Acta Cryst. A24, 321-324.

Egert, E. \& Sheldrick, G. M. (1985). Acta Cryst. A41, 262-268.

Gadol, S. M. \& DAvis, R. E. (1982). Organometallics, 1, 1607-1613.

Kunhne, M. E. \& Bornmann, W. G. (1989). J. Org. Chem. 54, $3407-3420$.

Kuehne, M. E., Zebovitz, T. C., Bornmann, W. G. \& Marko, I (1987). J. Org. Chem. 52, 4340-4349.
Kutney, J. P., Beck, J., Bylsma, F., Cook, J., Cretney, W. J., Fuj, K., Imhof, R. \& Treasurywala, A. M. (1975). Helv. Chim. Acta, 58, 1690-1719.

Langlois, N., Gueritte, F., Langlois, Y. \& Potier, P. (1976). $J$. Am. Chem. Soc. 98, 7017-7024.

Magnus, P., Stamford, A. \& Ladlow, M. (1991). J. Am. Chem. Soc. Submitted.

Moncrief, J. W. \& Lipscomb, W. N. (1965). J. Am. Chem. Soc. $87,4963-4964$.

Moncrief, J. W. \& Lipscomb, W. N. (1966). Acta Cryst. 21, 322-331.

PotIER, P. (1980). J. Nat. Prod. 43, 72-86.

SHELDRICK, G. M. (1988). SHELXTL-Plus88. Nicolet Instrument Corporation, Madison, Wisconsin, USA.

Stewart, R. F., Davidson, E. R. \& Simpson, W. T. (1965). J. Chem. Phys. 42, 3175-3187.

Acta Cryst. (1991). C47, 1566-1568

\title{
The Structure at $198 \mathrm{~K}$ of $(1 R, 5 R, 15 R, 16 R)-5$-Isopropenyl-2-methyl- 1 -[ $N$-(trans-2-phenylcyclohexyloxycarbonyl)amino]-2-cyclohexene
}

\author{
By Vincent M. Lynch, H. Kenan Yaser, James K. Whitesell and Brian E. Davis \\ Department of Chemistry and Biochemistry, University of Texas at Austin, Austin, TX 78712, USA
}

(Received 3 October 1990; accepted 2 January 1991)

Abstract. trans-2-Phenylcyclohexyl $N$-(5-isopropenyl2-methyl-2-cyclohexan-1-yl)carbamate, $\mathrm{C}_{23} \mathrm{H}_{31} \mathrm{NO}_{2}$, $M_{r}=353.50$, orthorhombic, $\quad P 2_{1} 2_{1} 2_{1}, \quad a=$ $8.813(2), \quad b=9.043(2), \quad c=25.643(5) \AA, \quad V=$ $2043.6(8) \AA^{3}, \quad Z=4, \quad D_{x}=1.15 \mathrm{~g} \mathrm{~cm}^{-3} \quad(198 \mathrm{~K})$, Mo $K \alpha$ radiation, $\lambda=0.7107 \AA, \mu=0.6734 \mathrm{~cm}^{-1}$, $F(000)=768, \quad T=198 \mathrm{~K}, \quad R=0.0547$ for 1772 reflections $\left[F_{o} \geq 4 \sigma\left(F_{o}\right)\right]$. Molecules are $\mathrm{H}$-bonded into infinite columns parallel to $\mathbf{a}$. The $\mathrm{H}$ bond involves the $\mathrm{NH}$ group and the carbonyl $\mathrm{O}$ atom of the carbamate moiety with relevant parameters: $\mathrm{N} 11-\mathrm{H} 11 \cdots \mathrm{O} 13$ (related by $\frac{1}{2}+x, \frac{1}{2}-y,-z$ ); $\mathrm{N} \cdots \mathrm{O}$

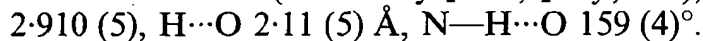

Experimental. The carbamate (1) was prepared from the adduct formed from $(R)$-limonene with the $N$ sulfinylcarbamate of $(1 R)$-trans-2-phenylcyclohexanol (Whitesell \& Carpenter, 1987; Whitesell, Carpenter, Yaser \& Machajewski, 1990) by reaction with hexamethyldisilazane followed by thermal rearrangement (Yaser, 1990). X-ray structural analy-

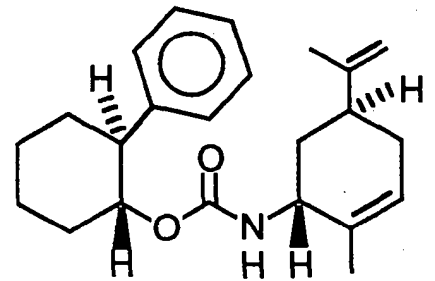

(1)

0108-2701/91/071566-03\$03.00 sis was used to confirm the stereochemistry of the chiral center bearing nitrogen (C1) and independently confirmed by internal comparison with the absolute stereochemistry of the trans-2phenylcyclohexanol used (Whitesell \& Lawrence, 1986). Crystals were obtained by slow evaporation from hexanes. The data crystal was a clear, colorless needle of approximate dimensions $0.11 \times 0.11 \times$ $0.29 \mathrm{~mm}$; the data were collected on a Nicolet $R 3$ diffractometer using a graphite monochromator and a Nicolet LT-2 low-temperature delivery system; lattice parameters were obtained from the least-squares refinement of 40 reflections with $20.2<2 \theta<23 \cdot 5^{\circ}$; $\omega$ scan technique with a $2 \theta$ range from $4 \cdot 0-52 \cdot 5^{\circ}$ and a $1 \cdot 2^{\circ} \omega$ scan at $5-10^{\circ} \min ^{-1}(h=-11 \rightarrow 11, k=-12$ $\rightarrow 12, l=0 \rightarrow 32$ ). Two symmetry equivalent octants of data were collected $(h k l$ and $-h,-k, l)$ yielding a total of 4788 reflections of which 2417 were unique $\left(R_{\text {int }}=0.0198\right)$; four reflections $(11 \overline{4}, \overline{1} \overline{3} \overline{2}, \overline{1} 24,125)$ were remeasured every 96 reflections to monitor instrument and crystal stability; a smoothed curve of the intensities of these check reflections was used to scale the data; the scaling factor ranged from 0.99121.014; the data were also corrected for Lp effects and absorption (based on crystal face measurements; transmission factor range was from 0.9832-0.9866). The data reduction, absorption and decay correction were applied using the Nicolet XRD SHELXTL-Plus software package (Sheldrick, 1988); reflections having $F_{o}<4 \sigma\left(F_{o}\right)$ were considered unobserved (645

(C) 1991 International Union of Crystallography 